\title{
DELINEAMENTO DO PERFIL CONCEITUAL DE AVALIAÇÃO DE MESTRANDOS EM ENSINO
}

http://dx.doi.org/ 10.5902/2318133839681

\author{
Simone Luccas ${ }^{1}$ \\ Marlize Spagolla Bernardelli² \\ João Coelho Neto ${ }^{3}$ \\ Lucken Bueno Lucas ${ }^{4}$
}

\begin{abstract}
Resumo
O conceito de avaliação é um tema relevante, polêmico e, ao mesmo tempo, controverso. Esse tema tem sido discutido no âmbito acadêmico, contudo, não se observa a presença dessas reflexões na prática educacional da sala de aula, sobretudo na educação básica. A discussão levanta a necessidade de uma mudança de concepção de avaliação, tendo em vista que as práticas avaliativas têm assumido uma postura classificatória e de seleção, aplicada num momento final da ação didático-pedagógica. Nesse contexto, procurou-se identificar o perfil conceitual da concepção de avaliação que profissionais em capacitação stricto sensu, em nível de Mestrado, apresentam. Após análise dos dados oriundos de uma avaliação diagnóstica realizada pela turma de 2016, identificou-se quatro zonas: realista, relacional, reflexiva e axiológica, sendo que a realista foi a dominante entre todas.
\end{abstract}

Palavras-chave: avaliação; perfil conceitual; práticas avaliativas.

\section{DELINEATION OF THE MASTER'S STUDENTS ASSESSMENT CONCEPTUAL PROFILE IN A TEACHING PROGRAM}

\begin{abstract}
The concept of Assessment is a relevant, polemic and at the same time controversial. This issue has been widely discussed in the scientific, however, the presence of these reflections in the educational practice of the classroom is not observed, especially in Basic Education. The discussion raises the need for a change in the assessment conception, in view of the fact that for many years, nowadays the assessment practices have assumed a classificatory posture and of the selection, applied in a final moment of the didactic-pedagogical action. In this context, it was sought to identify the conceptual profile of the assessment conception that professionals in training in a Graduate Program presents in the Master level. After analyzing the data from a diagnostic assessment performed by the class of 2016, four zones were identified: Realistic, Relational, Reflective and Axiological, and Realist was the dominant one among all.

Key-words: assessment; conceptual profile; assessment practices.
\end{abstract}

\footnotetext{
${ }^{1}$ Universidade Estadual do Norte do Paraná, campus Cornélio Procópio, Brasil. E-mail: simoneluccas@uenp.edu.br.

2 Universidade Estadual do Norte do Paraná, campus Cornélio Procópio, Brasil. E-mail: marlizespagolla@uenp.edu.br.

3 Universidade Estadual do Norte do Paraná, campus Cornélio Procópio, Brasil. E-mail: joaocoelho@uenp.edu.br.

${ }^{4}$ Universidade Estadual do Norte do Paraná, campus Cornélio Procópio, Brasil. E-mail: luckenlucas@uenp.edu.br. \begin{tabular}{|l|l|l|l|l|l}
\hline Regae: Rev. Gest. Aval. Educ. & Santa Maria & v. 8 & n. 17 & Pub. contínua 2019 & p. 1-12
\end{tabular}
} 


\section{Introdução}

avaliação apresenta-se como um tema de grande relevância para o âmbito $\triangle$ educacional, com diversas pesquisas a respeito (Hadji, 2001; Barlow, 2006; Luckesi, 2011; Esteban, 2003; Barriga, 2003; Buriasco, 1999, 2000; Sanmarti, 2011, Hoffmann, 1994, 2014; Roldão; Ferro, 2015). Muitos pesquisadores defendem a necessidade de uma mudança de entendimento sobre a avaliação e, consequentemente, da prática avaliativa, pois argumentam que a avaliação tem sido aplicada nos momentos finais da ação pedagógica, sempre num sentido classificatório e seletivo.

Segundo os autores supracitados, há décadas pesquisas indicam esse quadro, o que nos sugere questionar: por que ele se mantém? $O$ que faz com que a ação pedagógica se sirva de um paradigma sentencioso e classificatório de avaliação?

Hoffmann (1994) argumenta que a avaliação constitui características reprodutivas, ou seja, a prática avaliativa existente nas licenciaturas e cursos de magistério tem sido assumida pelos egressos desses cursos e praticada por eles em suas aulas, no decorrer de sua vida profissional. A autora comenta que a maioria dos professores reproduzem um modelo classificatório e seletivo: trabalham um conteúdo, prepararam prova, aplicam prova, corrigem prova, atribuem nota, registram notas.

Nesse sentido, Luckesi (2011) argumenta que

temos a habilidade de examinar, que herdamos tanto do sistema de ensino estabelecido e praticado ao longo dos anos como da nossa prática pessoal como educandos sucessivamente submetidos às práticas examinativas dos educadores que nos acompanharam em nossa trajetória de estudantes. Hoje, como educadores, repetimos com nossos educandos o que aconteceu conosco. (p. 30)

Considerando a problemática apresentada, de que o processo avaliativo praticado por muitos docentes está mais próximo de uma noção classificatória e seletiva do aluno e não de um acompanhamento dos processos de ensino e de aprendizagem, neste trabalho propõe-se a investigar o perfil conceitual de um grupo de estudantes, de um programa de pós-graduação stricto sensu, nível de mestrado da área de Ensino acerca de suas ideias sobre a avaliação no âmbito escolar.

O objetivo consistiu em identificar e evidenciar o perfil conceitual dos referidos mestrandos, por meio da análise das avaliações diagnósticas que os mesmos realizaram na fase inicial de uma disciplina sobre avaliação e prática docente.

\section{Aporte teórico}

Segundo Luckesi (2011), o papel fundamental da avaliação é diagnosticar o desenvolvimento do aluno, identificando seus pontos fortes e fracos para, a partir de então, intervir no processo de ensino, com vistas à promoção da aprendizagem, pois "o ato de avaliar tem como função investigar a qualidade do desempenho dos estudantes, tendo em vista proceder a uma intervenção para a melhoria dos resultados, caso seja necessária. Assim, a avaliação é diagnóstica" (p. 62).

Buriasco (2000) argumenta que, para além de mensurar o que os alunos não sabem, a avaliação deve ressaltar o que eles são capazes e sabem fazer. A autora destaca que o processo avaliativo deve compor a atividade escolar exercendo, sobretudo, 
sua função reguladora dos processos de ensino e de aprendizagem, oportunizando a todos os envolvidos os subsídios necessários para orientar a ação docente, bem como a compreensão do processo de aprendizagem e desenvolvimento do pensamento do aluno. Para a pesquisadora, a função da avaliação consiste em auxiliar na construção de uma aprendizagem satisfatória. Nesse sentido Buriasco (1999), argumenta que

avaliar pressupõe definir princípios em função de objetivos que se pretendem alcançar; estabelecer instrumentos para a ação e escolher caminhos para essa ação; verificar constantemente a caminhada, de forma crítica, levando em conta todos os elementos envolvidos no processo. Sendo assim, ela não possui uma finalidade em si, mas sim subsidia o curso de uma ação que visa construir um resultado previamente definido. (p. 72).

Hoffmann (1994) defende a perspectiva da avaliação mediadora, cujo sentido é avaliar de modo mais reflexivo e desafiador, favorecendo a relação entre o professor e os alunos, cujo saber ensinado é enriquecido a partir da compreensão dos saberes estudados. Essa perspectiva se opõe ao paradigma sentencioso e classificatório, pautado no modelo transmitir-verificar-registrar.

A autora enfatiza que a avaliação não deve ocorrer num momento final do trabalho educativo, mas ao longo do processo, no qual o professor sempre deve identificar o que foi compreendido pelos alunos. Hoffmann (1994) defende a existência da avaliação mediadora que conceba

o conhecimento como apropriação do saber pelo aluno e também pelo professor, como ação-reflexão-ação que se passa na sala de aula em direção a um saber aprimorado, enriquecido, carregado de significados, de compreensão. Dessa forma, a avaliação passa a exigir do professor uma relação epistemológica com o aluno - uma conexão entendida como reflexão aprofundada a respeito das formas como se dá a compreensão do educando sobre o objeto do conhecimento. (p. 56)

Para Rosa, Coutinho e Flores (2017) o entendimento que os alunos têm da avaliação determina o modo como aprendem, isto é, a operacionalização da avaliação implica na motivação e na aprendizagem dos alunos. Em outras palavras, a avaliação pode ser vista como um meio para a aprendizagem (Flores et al., 2014). As pesquisadoras evidenciam que a avaliação constitui um elemento crucial do currículo que, por seu turno, necessita de renovação: "Alguns autores chamam a atenção para a necessidade de os sistemas educacionais precisarem ser cada vez mais criativos no que diz respeito às práticas de avaliação adotadas bem como para a importância da sua renovação" (Rosa; Coutinho; Flores, 2016, p 418).

Assim, esta pesquisa apresenta os resultados de uma investigação acerca do perfil conceitual de um grupo de mestrandos que cursam a disciplina de Avaliação e Prática Docente, quando da realização de uma avaliação diagnóstica realizada sobre a temática avaliação e seu papel nos processos de ensino e de aprendizagem.

No que se refere ao perfil conceitual, Mortimer (1994, 1995, 2000) o defende como sendo uma forma de delineamento da variedade de pensamentos e linguagens desenvolvidos. Esse modelo de perfil proposto teve como foco, nesta pesquisa, a análise da concepção dos participantes da pesquisa em relação ao tema avaliação 
Mortimer (2000) define perfil conceitual como "um sistema supra-individual de formas de pensamento que pode ser atribuído a qualquer indivíduo dentro de uma mesma cultura" (p. 80). Segundo ele a construção de novos conceitos não pressupõe o abandono das concepções prévias, mas a tomada de consciência do contexto em que elas são aplicáveis, o que difere do modelo de mudança conceitual. A defesa é que existem visões heterogêneas de um mesmo conceito, que coexistem no âmbito acadêmico e escolar, e que os diferentes perfis se complementam.

A ideia fundamental do perfil conceitual é de que o desenvolvimento de um conceito dá origem a uma pluralidade de significados, decorrentes de diferentes formas de conhecimento, contextos e linguagens presentes nas esferas da vida acadêmica, escolar e social. O autor alega a relevância de se distinguir as características ontológicas e epistemológicas de cada zona do perfil conceitual para que o indivíduo tome consciência de seu próprio perfil pois, dessa forma, ele terá mais chance de privilegiar determinados mediadores e linguagens sociais.

A intenção dessa fundamentação teórica é contribuir para o delineamento do perfil conceitual que os mestrandos que cursam a disciplina obrigatória de Avaliação e Prática Docente apresentaram em uma avaliação diagnóstica realizada na disciplina, pois para Mortimer e El-Hani (2014) "o mundo em si não pode ser entendido inteiramente a partir de uma única perspectiva, mas sim interpretado por diferentes pontos de vista e até mesmo complementares" (p. 6). Além disso, para Sepulveda; Mortimer e El-Hani (2013) "os perfis conceituais, além de modelar a heterogeneidade do pensamento verbal, pretendem modelar os processos de gênese destes modos de pensar em determinados contextos sociais de produção de conhecimento" (p. 474).

Em se tratando de ensino e aprendizagem, Bernardelli (2014) aponta que,

nas últimas décadas, o ensino e a aprendizagem passaram por várias propostas como: ensino por descoberta, ensino por mudança conceitual, ensino por investigação, ensino baseado no perfil epistemológico, ensino baseado no perfil conceitual, todos vinculadas a educadores, dentre eles Piaget, Posner, Gil-Pérez, Bachelard e Mortimer na perspectiva de possíveis entendimentos a respeito da aprendizagem em Ciências. O perfil conceitual é uma perspectiva dessas propostas, como uma forma para delinear a variedade de pensamentos e linguagens existentes em uma sala de aula. (p. 57)

Nessa perspectiva, questões da aprendizagem e do ensino da ciência podem ser consideradas de uma nova forma, tendo como base o perfil conceitual, visto que é possível ensinar um conceito em um certo nível do perfil sem referência a um nível menos complexo, uma vez que são epistemologicamente e ontologicamente diferentes. Nesse sentido, o processo de aprendizagem pode ser pensado como a construção de um corpo de noções baseadas em novos fatos e experimentos apresentados aos alunos no processo de ensino. O novo conceito não depende necessariamente dos anteriores e poderia ser aplicado a um novo domínio diferente (Mortimer, 1993). 
Assim, Mattos (2012) argumenta que a abordagem do perfil conceitual está comprometida com a ideia de que diferentes modos de pensar sócio-historicamente construídos sobre um conceito coexistem em um indivíduo, mesmo após a educação formal. Do ponto de vista educacional, os estudantes não necessariamente abandonam suas antigas concepções quando aceitam as novas.

Portanto, como o perfil conceitual é composto de diferentes zonas que o representam a maneira de pensar, falar, usar um conceito. Um indivíduo pode absorver uma nova zona ou alterar as mais antigas em seus perfis conceituais, à vista disso, também se torna consciente dessa diversidade de modos de pensar sobre o conceito e seus domínios distintos de aplicação. As zonas de perfil conceitual podem coexistir no mesmo indivíduo, incluindo os modos de pensamento não científico.

\section{Abordagem metodológica}

A pesquisa foi definida como qualitativa, de cunho interpretativo. A coleta de dados ocorreu no primeiro dia de aula da disciplina de Avaliação e Prática Docente de um curso de Mestrado Profissional em Ensino, da Universidade Estadual do Norte do Paraná, campus Cornélio Procópio. Foi realizada uma avaliação diagnóstica para levantar as noções prévias que os mestrandos apresentavam sobre a temática avaliação, com a intenção de delinear o perfil conceitual do assunto.

A avaliação diagnóstica continha três questões: questão 1: o que é avaliação para você? Questão 2: seria possível você trabalhar uma disciplina escolar sem realizar qualquer avaliação? Questão 3: O que você espera desta disciplina? Neste artigo, serão analisadas as duas primeiras questões, já que o foco não é analisar a disciplina em si, mas evidenciar o perfil conceitual dos mestrandos.

A análise desse perfil ocorreu em três etapas: levantamento individual de aspectos relevantes das respostas dos mestrandos; levantamento dos aspectos relevantes da turma; e, estabelecimento de categorias gerais e de zonas conceituais.

\section{Delineamento do perfil conceitual: análise dos dados}

De posse das avaliações diagnósticas o primeiro passo foi analisar as duas primeiras questões dos participantes, procurando identificar as sentenças ou termos relevantes das respostas de cada deles.

Quadro 1 -

Sentenças e termos individuais dos participantes da pesquisa.

\begin{tabular}{|l|l|}
\hline Mestrando & \multicolumn{1}{|c|}{ Sentenças e termos } \\
\hline \multirow{3}{*}{ M1 } & - Diagnóstico de pontos positivos e negativos; \\
& - A avaliação está relacionada com os processos de ensino e de \\
& aprendizagem; \\
& - Análise dos procedimentos da aula; \\
& - Capacidade cognitiva; \\
& - Reflexão da prática docente. \\
\hline M2 & - Identifica a evolução do professor e do aluno; \\
& - Fornece subsídios para próximas aulas. \\
\hline M3 & - Interfere na aprendizagem; \\
\hline
\end{tabular}




\begin{tabular}{|c|c|}
\hline & $\begin{array}{l}\text { - É subjetivo; } \\
\text { - Envolve valores pessoais; } \\
\text { - Ética e equilíbrio. }\end{array}$ \\
\hline M4 & $\begin{array}{l}\text { - Processo de valorização; } \\
\text { - Mais de um instrumento avaliativo; } \\
\text { - Envolve processo de ensino e de aprendizagem. }\end{array}$ \\
\hline M5 & $\begin{array}{l}\text { - Reflexão; } \\
\text { - Envolve processo de ensino e de aprendizagem; } \\
\text { - Deve ser constante; } \\
\text { - Deve ser diversificada; } \\
\text { - Destinada a analisar professor e aluno. }\end{array}$ \\
\hline M6 & $\begin{array}{l}\text { - Reflexão para o professor autoavaliar-se; } \\
\text { - Assimilação do conhecimento. }\end{array}$ \\
\hline M7 & $\begin{array}{l}\text { - Medir conhecimento do aluno; } \\
\text { - Angústia do professor; } \\
\text { - Avaliação acontece a todo momento; } \\
\text { - Procedimentos e habilidades. }\end{array}$ \\
\hline M8 & $\begin{array}{l}\text { - Medir conhecimento do aluno mediante provas; } \\
\text { - Trabalhar com planos e metas; } \\
\text { - Participação e desenvolvimento do aluno. }\end{array}$ \\
\hline M9 & $\begin{array}{l}\text { - Método. } \\
\text { - Verificação do entendimento do aluno. }\end{array}$ \\
\hline M10 & $\begin{array}{l}\text { - Método; } \\
\text { - Averiguar a aprendizagem do aluno; } \\
\text { - Caráter formativo para aluno e professor; } \\
\text { - Avalia-se a todo instante. }\end{array}$ \\
\hline M11 & $\begin{array}{l}\text { - Processo contínuo; } \\
\text { - Verificar condições e habilidades do aluno; } \\
\text { - Possibilita ao professor repensar sua prática; } \\
\text { - Possibilita repensar a gestão de conteúdo e de classe; } \\
\text { - Tudo na vida é avaliado. }\end{array}$ \\
\hline M12 & $\begin{array}{l}\text { - Verificar a aprendizagem; } \\
\text { - Verificar a aprendizagem de conteúdos, valores, socialização, } \\
\text { crescimento humano; } \\
\text { - Averiguar o trabalho do professor; } \\
\text { - Avaliação auxilia o professor no desempenho de seu trabalho e a } \\
\text { organizar seus métodos de trabalho. }\end{array}$ \\
\hline M13 & $\begin{array}{l}\text { - Verificação da aprendizagem; } \\
\text { - Avaliar o trabalho do professor para ver se atingiu seus objetivos; } \\
\text { - Ocorre constantemente, é contínua. }\end{array}$ \\
\hline
\end{tabular}

Fonte: dados da pesquisa. 
Após o levantamento das 46 sentenças e termos destacados das respostas individuais dos mestrandos foi realizado um agrupamento de sentenças ou termos que apresentavam o mesmo sentido, bem como a análise da frequência com que eles ocorreram. Foram configurados oito agrupamentos de mesmo sentido.

$\mathrm{Na}$ segunda etapa foi feita uma análise grupal. Assim, as categorias gerais começam a se constituir, tendo em vista que elas dão sustentação para 0 estabelecimento das zonas conceituais.

Quadro 2 -

Levantamento dos aspectos relevantes da turma.

\begin{tabular}{|c|c|c|}
\hline Sentenças/termos & Frequência absoluta & Fonte dos dados \\
\hline $\begin{array}{l}\text {-Verificar a aprendizagem do } \\
\text { aluno } \\
\text {-Averiguar } \\
\text {-Identificar } \\
\text {-Diagnosticar } \\
\text {-Método } \\
\text {-Medir conhecimento }\end{array}$ & 13 & $\begin{array}{l}\text { M1, M2, M7, M8, M9, M10, M12, } \\
\text { M13 }\end{array}$ \\
\hline $\begin{array}{lll}\text {-Verificar o trabalho do } \\
\text { professor } & & \\
\text { - Averiguar } & & \\
\text { - Identificar } & & \\
\text {-Diagnosticar } & & \end{array}$ & 03 & $\mathrm{M} 2, \mathrm{M} 12, \mathrm{M} 13$ \\
\hline $\begin{array}{l}\text {-Reflexão da prática } \\
\text { docente/Análise }\end{array}$ & 09 & $\begin{array}{l}\text { M1, M2, M5, M6, M7, M8, M11, } \\
\text { M12 }\end{array}$ \\
\hline $\begin{array}{l}\text { Processo de ensino e de } \\
\text { aprendizagem }\end{array}$ & 05 & M1, M4, M5, M10 \\
\hline Processo de aprendizagem & 06 & M1, M3, M6, M7, M8, M11 \\
\hline $\begin{array}{l}\text { Instrumentos avaliativos } \\
\text { (diversificado, provas) }\end{array}$ & 03 & M4, M5, M8 \\
\hline $\begin{array}{l}\text { Avaliação } \\
\text { contínua/constante }\end{array}$ & 05 & M5, M7, M10, M11, M13 \\
\hline $\begin{array}{l}\text { Caráter subjetivo (valores } \\
\text { pessoais, ética e equilíbrio) }\end{array}$ & 02 & M3, M4 \\
\hline
\end{tabular}

Fonte: dados da pesquisa.

Na terceira etapa os agrupamentos definidos foram reorganizados em dez unidades, as quais integraram seis categorias gerais que, por sua vez, constituíram as quatro zonas conceituais, como apresentado no quadro 3. 
Quadro 3 -

Quadro geral das unidades, categorias e zonas conceituais.

\begin{tabular}{|l|l|l|}
\hline Unidades & Categorias & Zonas Conceituais \\
\cline { 1 - 2 } Aprendizagem do aluno & \multirow{2}{*}{ Método de verificação } & \\
\cline { 1 - 2 } Trabalho do professor & Instrumento avaliativo & Realista \\
\cline { 1 - 2 } Proversificado & $\begin{array}{l}\text { Periodicidade da } \\
\text { avaliação }\end{array}$ & \\
\cline { 1 - 2 } Contínua & Relação com processos & Relacional \\
\cline { 1 - 2 } Aprendizagem & $\begin{array}{l}\text { Reflexão da prática } \\
\text { Ensino e aprendizagem }\end{array}$ & Reflexiva \\
\cline { 1 - 2 } $\begin{array}{l}\text { Reflexão e análise da prática } \\
\text { docente }\end{array}$ & Envolvimento de valores & Axiológica \\
\cline { 1 - 2 } Pessoais &
\end{tabular}

Fonte: dados da pesquisa.

Em síntese, o processo da análise caminhou das noções individuais para as coletivas, sendo levantadas inicialmente 46 sentenças ou termos destacados das respostas individuais dos mestrandos. Em seguida essas sentenças ou termos foram organizadas em oito grupos cuja sentença ou termo apresentasse o mesmo sentido, constituindo grupos de sentido do coletivo da sala.

Num momento posterior esses agrupamentos foram reorganizados em dez unidades, as quais integraram seis categorias gerais que, por sua vez, constituíram as quatro zonas conceituais, a saber: realista, relacional, reflexiva e axiológica.

A zona conceitual realista envolveu as categorias: método de verificação, instrumento avaliativo e periodicidade. Essa zona conceitual foi a que obteve maior frequência, tendo 26 sentenças ou termos classificados.

Foi interessante observar que as sentenças/termos relativos à verificação da aprendizagem dos alunos apresentaram alta frequência (13) em comparação com a verificação do trabalho do professor (3). Esse fato mostra que a avaliação é vista por muitos mestrandos como um meio de verificação somente da aprendizagem do aluno e não como um guia ou parâmetro para o desenvolvimento da ação pedagógica.

Em se tratando da categoria instrumento avaliativo, $10 \%$ das sentenças/termos fizeram uma relação direta entre avaliação e instrumentos de avaliação, ou seja, provas. Isso mostra que na concepção dos mestrandos avaliar significa preparar instrumentos adequados e diversificados. Segundo Hadji (2001) e Barlow (2006), esse constitui um tipo de avaliação realizada ao final de um estudo, denominada somativa. Para além deste há outros tipos de avaliação como a diagnóstica e a formativa, por exemplo.

Com relação à periodicidade com que as avaliações são realizadas $10 \%$ das sentenças/termos indicaram que a avaliação deve ser contínua e constante. Tal fato constitui-se um paradoxo, pois se deve ser constante, por que alguns professores as realizam poucas vezes durante um bimestre? 
A zona conceitual relacional abarcou uma categoria que envolveu a relação com o processo somente de aprendizagem ou de ensino e de aprendizagem. É importante observar que os mestrandos reconhecem que a avaliação está relacionada, tanto com o ensino, quanto com a aprendizagem. Nesta zona, onze sentenças/termos foram classificadas.

Já a zona conceitual reflexiva envolveu nove sentenças/termos. Essa quantidade correspondeu a $20 \%$ do total, revelando que $80 \%$ dos mestrandos não veem a avaliação como um meio para a reflexão de sua prática docente, ou seja, não a utilizam como avalição formativa, a qual tem justamente como foco guiar e orientar a ação pedagógica.

A zona conceitual axiológica apresentou somente duas sentenças/termos, indicando que poucos mestrandos relacionam a avaliação a valores pessoais e éticos. Esse é um campo fértil para novas pesquisas, tendo em vista que os valores e as condições de quem se avalia podem interferir no julgamento das atividades avaliativas, como apontam Carvalho e Gil-Perez (2011).

A zona conceitual realista foi dominante entre as demais delineadas. Isso mostra que $50 \%$ dos mestrandos veem a avaliação relacionada diretamente com a aprendizagem dos alunos, com instrumentos de avaliação e frequência das mesmas. Somente $6 \%$ das sentenças/termos analisados nessa zona indicaram verificar o trabalho do professor, ou seja, o ensino.

As demais zonas conceituais - relacional, reflexiva e axiológica - envolveram os $46 \%$ restantes das sentenças/termos analisados. Os teóricos do campo da avaliação apresentados na fundamentação teórica deste trabalho evidenciam que justamente essas três zonas é que devem ser mais exploradas e praticadas na ação pedagógica, ou seja, para além de avaliações de caráter somativo, faz-se necessário praticar avaliações de caráter formativo e diagnóstico.

\section{Considerações finais}

O presente trabalho objetivou empreender uma reflexão acerca das concepções avaliativas de um grupo de estudantes de mestrado. Pesquisadores como Luckesi (2001), Buriasco (1999, 2000), Hoffmann (1994, 2014), entre outros, argumentam que embora tenham se passado décadas dos primórdios da ação de avaliar no meio educacional, a avaliação ainda se aproxima da ideia de exame, ocorrendo num momento final da ação pedagógica, sempre num sentido classificatório e seletivo e, sendo assim, defendem a necessidade de uma mudança de concepção de avaliação do processo de ensino e de aprendizagem que auxilie 0 docente em sua organização pedagógica e 0 aluno na administração de sua aprendizagem.

Nesse sentido, a proposta deste trabalho foi identificar o perfil conceitual da concepção de avaliação que profissionais em capacitação stricto sensu, em nível de Mestrado, apresentam.

Os participantes desta pesquisa, ao iniciarem uma disciplina de Avaliação e Prática Docente, de um curso Mestrado da área de Ensino, realizaram uma avaliação diagnóstica com o objetivo de levantar suas noções prévias de avaliação, possibilitando que fosse evidenciado o perfil conceitual da turma no tema tratado. 
Fundamentado teoricamente em trabalhos de pesquisadores do campo da avaliação escolar e de noções de perfil conceitual, fez-se a análise dos dados oriundos da avaliação diagnóstica e o delineamento do perfil conceitual da turma. Foram detectadas quatro zonas conceituais, a saber: realista, relacional, reflexiva e axiológica, sendo que a zona realista foi a dominante entre todas.

As zonas conceituais relacional, reflexiva e axiológica que estão menos presentes nos perfis conceituais devem ser mais exploradas e praticadas na ação pedagógica. Compreende-se que somente com um acompanhamento frequente da ação pedagógica, seja por meio da avaliação diagnóstica ou formativa, é que se pode guiar o ensino e a aprendizagem do conhecimento escolar.

Para um trabalho futuro, pretende-se analisar atividades dos mestrandos durante 0 curso na expectativa de investigar se a concepção de avaliação sofreu modificações, e quais seriam essas modificações, ou seja, analisar se ocorreu uma ampliação do perfil conceitual delineado neste trabalho.

\section{Referências}

BARLOW, Michel. Avaliação escolar: mitos e realidades. Porto Alegre: Artmed, 2006.

BARRIGA, Ángel Diaz. Uma polêmica em relação ao exame. In: ESTEBAN, Maria. Teresa. Avaliação: uma prática em busca de novos sentidos. Rio de Janeiro: DP\&A, 2003, p. 51-82.

BERNARDELLI, Marlize Spagolla. A interdisciplinaridade educativa na contextualização do conceito de Transformação Química em um curso de Ciências Biológicas. Londrina: UEL, 2014. 218f. Tese (doutorado em Ensino de Ciências e Educação Matemática). Programa de Pós-Graduação em Ensino de Ciências e Educação Matemática, Universidade Estadual de Londrina.

BURIASCO, Regina Luzia Corio. Algumas considerações sobre avaliação educacional. Revista: Estudos em avaliação educacional, 2000, n. 22, p 155-178.

BURIASCO, Regina Luzia Corio. Avaliação em matemática: um estudo das respostas de alunos e professores. Marília: Unesp, 1999. 238f. Tese (doutorado em Educação). Programa de Pós-Graduação em Educação, Universidade Estadual Paulista.

CARVALHO, Anna Maria. Pessoa; GIL-PEREZ, Daniel. Formação de professores de ciências: tendências e inovações. São Paulo: Cortez, 2011.

ESTEBAN, Maria Teresa. Avaliação: uma prática em busca de novos sentidos. Rio de Janeiro: DP\&A, 2003.

FLORES, Maria Assunção; VEIGA SIMÃO, Ana Margarida; BARROS, Alexandra, PEREIRA, Diana. Perceptions of effectiveness, fairness and feedback of assessment methods: a study in higher education, Studies in Higher Education. International Journal of Engineering Education, v. 29, n. 4, 2014, p. 940-947.

HADJI, Charles. A avaliação desmistificada. Porto Alegre: Artmed, 2001.

HOFFMANN, Jussara Maria Lerch. Avaliação mediadora: uma relação dialógica na construção do conhecimento. São Paulo: FDE, 1994.

HOFFMANN, Jussara Maria Lerch. Avaliar para promover: as setas do caminho. Porto Alegre: Mediação, 2014. 
LUCKESI, Cipriano Carlos. Avaliação da aprendizagem escolar: estudos e proposições. São Paulo: Cortez, 2011.

MATTOS, Cristiano Rodrigues. Conceptual profile as a model of a complex world. In: MORTIMER, Eduardo Fleury; EL-HANI, Charbel Niño (eds.). Conceptual profile: a theory of teaching and learning scientific concepts. Contemporary Trends and Issues in Science Education Series, v. 43, Berlin: Springer. 2012.

MORTIMER, Eduardo Fleury. Linguagem e formação de conceitos no ensino de ciências. Belo Horizonte: UFMG, 2000.

MORTIMER, Eduardo Fleury. Evolução do atomismo em sala de aula: mudança de perfis conceituais. São Paulo: USP, 1994. 281f. Tese (doutorado em Educação). Faculdade de Educação, Universidade de São Paulo.

MORTIMER, Eduardo Fleury. Studying conceptual evolution in the classroom as conceptual profile change. In: The Proceedings of the Third International Seminar on Misconceptions and Educational Strategies in Science and Mathematics, Misconceptions. Trust: Ithaca, 1993, p. 1-50.

MORTIMER, Eduardo Fleury. Concepções atomísticas dos estudantes. Química Nova na Escola. 1995, p. 23-26. Disponível em http://qnesc.sbq.org.br/online/qnesc01/aluno.pdf. Acesso em 22 set. 2016.

MORTIMER, Eduardo Fleury; EL-HANI, Charbel (eds.). Conceptual profiles: a theory of teaching and learning scientific concepts, Springer, 2014.

ROLDÃO, Maria do Céu; FERRO, Nuno. O que é avaliar? Reconstrução de práticas e concepções de avaliação. Estudos em Avaliação Educacional. São Paulo, v. 26, n. 63, 2015, p. 570-594.

ROSA, Selma dos Santos; COUTINHO, Clara Pereira; FLORES, Maria Assunção. Online peer assessment no ensino superior: uma revisão sistemática da literatura em práticas educacionais. Avaliação: Revista da Avaliação da Educação Superior. Campinas, v. 22, 2017, p. 55-83.

ROSA, Selma dos Santos; COUTINHO, Clara Pereira; FLORES, Maria Assunção. Online peer assessment: method and digital technologies. INTERNATIONAL CONFERENCE ON HIGHER EDUCATION ADVANCES, 2, 2016. Actas ... Valencia, 2016, p. 418-423.

SANMARTí, Neus. Evaluar para aprender, evaluar para calificar. In: CAÑAL, Pedro. Didactica de la Biologia y la Geologia. Espanha: Grao, 2011, p. 151-171.

SEPÚLVEDA, Claudia; MORTIMER, Eduardo Fleury; EL-HANI, Charbel N. Construção de um perfil conceitual de adaptação: implicações metodológicas para o programa de pesquisa sobre perfis conceituais e o ensino de evolução. Investigações em Ensino de Ciências, v. 18, n. 2, 2013, p. 439-479.

Simone Luccas é professora no Programa de Pós-Graduação em Ensino e no Centro de Ciências Humanas e da Educação da Universidade Estadual do Norte do Paraná.

Orcid: https://orcid.org/0000-0002-5435-5478.

Endereço: PR 160, Km 0 - 86300-000 - Cornélio Procópio - PR - Brasil.

E-mail: simoneluccas@uenp.edu.br. 
Marlize Spagolla Bernardelli é professora no Programa de Pós-Graduação em Ensino da Universidade Estadual do Norte do Paraná.

Orcid: https://orcid.org/0000-0001-7377-7804.

Endereço: PR 160, Km 0 - 86300-000 - Cornélio Procópio - PR - Brasil.

E-mail: marlizespagolla@uenp.edu.br.

Joao Coelho Neto é professor no Programa de Pós-Graduação em Ensino e no Centro de Ciências Humanas da Educação da Universidade Estadual do Norte do Paraná.

Orcid: http://orcid.org/0000-0002-6154-3266.

Endereço: PR 160, Km 0 - 86300-000 - Cornélio Procópio - PR - Brasil.

E-mail: joaocoelho@uenp.edu.br.

Lucken Bueno Lucas é professor no Programa de Pós-Graduação em Ensino e no Centro de Ciências Humanas e da Educação da Universidade Estadual do Norte do Paraná.

Orcid: https://orcid.org/0000-0003-2122-8672.

Endereço: PR 160, Km 0 - 86300-000 - Cornélio Procópio - PR - Brasil.

E-mail: luckenlucas@uenp.edu.br.

Recebido em 23 de julho de 2019.

Aceito em 8 de setembro de 2019.

(c) (i) 